\title{
Feller property of the multiplicative coalescent with linear deletion
}

\author{
BALÁZS RÁTH
}

Department of Stochastics, Budapest University of Technology and Economics, 1 Egry József u., 1111 Budapest, Hungary.E-mail: rathb@math.bme.hu

We modify the definition of Aldous' multiplicative coalescent process (Ann. Probab. 25 (1997) 812-854) and introduce the multiplicative coalescent with linear deletion (MCLD). A state of this process is a squaresummable decreasing sequence of cluster sizes. Pairs of clusters merge with a rate equal to the product of their sizes and clusters are deleted with a rate linearly proportional to their size. We prove that the MCLD is a Feller process. This result is a key ingredient in the description of scaling limits of the evolution of component sizes of the mean field frozen percolation model (J. Stat. Phys. 137 (2009) 459-499) and the so-called rigid representation of such scaling limits (Electron. J. Probab. To appear).

Keywords: Feller process; multiplicative coalescent

\section{Introduction}

Let us define

$$
\begin{aligned}
& \ell_{\infty}^{\downarrow}=\left\{\underline{m}=\left(m_{1}, m_{2}, \ldots\right): m_{1} \geq m_{2} \geq \cdots \geq 0\right\}, \\
& \ell_{2}^{\downarrow}=\left\{\underline{m} \in \ell_{\infty}^{\downarrow}: \sum_{i=1}^{\infty} m_{i}^{2}<\infty\right\}, \\
& \ell_{0}^{\downarrow}=\left\{\underline{m} \in \ell_{\infty}^{\downarrow}: \exists i_{0} \in \mathbb{N}: m_{i}=0 \text { for any } i \geq i_{0}\right\} .
\end{aligned}
$$

For $\underline{m}, \underline{m}^{\prime} \in \ell_{2}^{\downarrow}$ one defines the distance

$$
\mathrm{d}\left(\underline{m}, \underline{m}^{\prime}\right)=\left\|\underline{m}-\underline{m}^{\prime}\right\|_{2}=\left(\sum_{i \geq 1}\left(m_{i}-m_{i}^{\prime}\right)^{2}\right)^{1 / 2} .
$$

The metric space $\left(\ell_{2}^{\downarrow}, \mathrm{d}(\cdot, \cdot)\right)$ is complete and separable.

The multiplicative coalescent process (or briefly MC process), defined in [3], Section 1.5, is a continuous-time Markov process $\mathbf{m}_{t}, t \geq 0$ with state space $\ell_{2}^{\downarrow}$. The state $\mathbf{m}_{t}$ represents the ordered sequence of sizes of components, where two components of size $m_{i}$ and $m_{j}$ merge with rate $m_{i} \cdot m_{j}$. By [3], Proposition 5, the multiplicative coalescent process has the Feller property with respect to the metric $\mathrm{d}(\cdot, \cdot)$ on $\ell_{2}^{\downarrow}$. On the other hand, if $\mathbf{m}_{0} \in \ell_{\infty}^{\downarrow} \backslash \ell_{2}^{\downarrow}$, then all of the components instantaneously coagulate and form one component with infinite mass, see [14], Section 2.1. In Section 2, we collect the basic results about MC relevant for our study. 
Let $\lambda \in \mathbb{R}_{+}$. For any $\underline{m} \in \ell_{2}^{\downarrow}$ we want to define a continuous time Markov process $\mathbf{m}_{t}$ with state space $\ell_{2}^{\downarrow}$ where $\mathbf{m}_{0}=\underline{m}$ and $\mathbf{m}_{t}$ represents the ordered sequence of sizes of components of a coagulation-deletion process at time $t$. We want the dynamics of the process $\mathbf{m}_{t}, t \geq 0$ to satisfy

(i) two components of size $m_{i}$ and $m_{j}$ merge with rate $m_{i} \cdot m_{j}$,

(ii) a component of size $m_{i}$ is deleted with rate $\lambda \cdot m_{i}$.

We are going to call such a process a multiplicative coalescent with linear deletion with deletion rate $\lambda$, and briefly denote it by $\operatorname{MCLD}(\lambda)$.

If $\underline{m} \in \ell_{0}^{\downarrow}$, then the $\operatorname{MCLD}(\lambda)$ process obviously exists and $\mathbf{m}_{t} \in \ell_{0}^{\downarrow}$ for any $t \geq 0$. In fact, if $\underline{m} \in \ell_{\infty}^{\downarrow}$ with $\sum_{i=1}^{\infty} m_{i}<\infty$ then the definition of $\operatorname{MCLD}(\lambda)$ is still quite simple because the time between consecutive coalescences/deletions is always positive. On the other hand, for initial conditions with infinite total mass, the set of times when a coalescence or deletion occurs will be dense in $\mathbb{R}_{+}$, and it is not a priori clear that a well-defined stochastic process satisfying (1.2) exists (see Remark 1.4 below for related non-existence results).

In Section 3, we will give a graphical construction of the process $\mathbf{m}_{t}$ with initial state $\underline{m} \in \ell_{2}^{\downarrow}$ and deletion rate $\lambda$. This construction of $\operatorname{MCLD}(\lambda)$ is similar to, but not as simple as the graphical construction of the MC given in [3], Section 1.5, because $\operatorname{MCLD}(\lambda)$ lacks the monotonicity properties of MC, see Remark 1.3 below. In Section 3 we also prove the following proposition.

Proposition 1.1. For any $\underline{m} \in \ell_{2}^{\downarrow}$ our graphical construction of $\operatorname{MCLD}(\lambda)$ (see Section 3) almost surely gives a function $t \mapsto \mathbf{m}_{t}$ with $\mathbf{m}_{0}=\underline{m}$ which is càdlàg with respect to the $\mathrm{d}(\cdot, \cdot)$-metric.

The main result of this paper is that our construction indeed gives rise to a well-behaved continuous-time Markov process on $\ell_{2}^{\downarrow}$ :

Theorem 1.2 (Feller property). Let $\underline{m}^{(n)}, n \in \mathbb{N}$ be a convergent sequence of elements of $\ell_{2}^{\downarrow}$ with limit $\underline{m}^{(\infty)}$, that is, $\lim _{n \rightarrow \infty} \mathrm{d}\left(\underline{m}^{(n)}, \underline{m}^{(\infty)}\right)=0$. For any $t \in \mathbb{R}_{+}$and $n \in \mathbb{N}_{+} \cup\{\infty\}$, denote by $\mathbf{m}_{t}^{(n)}$ the $\operatorname{MCLD}(\lambda)$ process with initial condition $\underline{m}^{(n)}$ at time $t$. For any $t \geq 0$ we have

$$
\mathbf{m}_{t}^{(n)} \stackrel{d}{\longrightarrow} \mathbf{m}_{t}^{(\infty)}, \quad n \rightarrow \infty
$$

where $\stackrel{d}{\longrightarrow}$ denotes convergence in distribution of random variables on the Polish space $\left(\ell_{2}^{\downarrow}, \mathrm{d}(\cdot, \cdot)\right)$.

We will prove Theorem 1.2 in Section 4 using an argument that involves truncation and coupling.

Remark 1.3. The reason why the proof of the Feller property for $\operatorname{MCLD}(\lambda)$ is more involved than the proof of the Feller property for MC (cf. the proof of [3], Proposition 5, in [3], Section 4.2) is that the natural graphical construction of $\operatorname{MCLD}(\lambda)$ is not monotone: 
If we obtain $\mathbf{m}_{t}^{\prime}, t \geq 0$ from $\mathbf{m}_{t}, t \geq 0$ by inserting an extra deletion event at time $t_{1}$, then it might happen that this deletion prevents later coagulations and deletions, so that $\mathbf{m}_{t_{2}}^{\prime}$ has more/bigger components than $\mathbf{m}_{t_{2}}$ for some $t_{2}>t_{1}$. Similarly, insertion of an extra coagulation event at some time might lead to the deletion of more/bigger components and thus create a state with fewer/smaller components at a later time.

\subsection{Motivation, related results}

Our reason for developing the theory of $\operatorname{MCLD}(\lambda)$ on the state space $\ell_{2}^{\downarrow}$ is that we want to understand the scaling limit of the time evolution of large connected component sizes in the self-organized critical mean field frozen percolation model [18], as we now explain.

The frozen percolation process on the binary tree was defined in [5]: the model is a modification of the dynamical percolation process on the binary tree which makes the following informal description precise: edges appear with rate 1 and if an infinite component appears, we immediately "freeze" it, and we do not allow edges with an end-vertex in a frozen component to appear.

Remark 1.4. I. Benjamini and O. Schramm showed that it is impossible to define a similar modification of the percolation process on $\mathbb{Z}^{2}$, cf. [23], Section 3, Remark (i). Various modifications of the two-dimensional frozen percolation model where large finite clusters are frozen are further explored in [12,20-22]. The result of [13] about the closely related model of two-dimensional self-destructive percolation implies non-existence of the so-called two dimensional forest fire process, cf. [13], Section 3.2. However, the result of [2] about self-destructive percolation on the high-dimensional lattice $\mathbb{Z}^{d}$ indicates that the self-organized critical forest fire process on $\mathbb{Z}^{d}$ should exist if $d$ is high enough. The existence and uniqueness of the subcritical forest fire process on $\mathbb{Z}^{d}$ was proved in $[10,11]$.

Let us now recall the notion of mean-field frozen percolation process from [18] (using slightly different notation).

Definition 1.5 $(\operatorname{FP}(n, \lambda(n)))$. We start with a graph $F_{0}^{(n)}$ on $n$ vertices. Between each pair of unconnected vertices an edge appears with rate $1 / n$; also, every connected component of size $k$ is deleted with rate $\lambda(n) \cdot k$. (When a component is deleted, its vertices as well as its edges are removed from the graph.) Let $F_{t}^{(n)}$ be the graph at time $t$. Denote by

$$
\mathbf{M}^{(n)}(t)=\left(M_{1}^{(n)}(t), M_{2}^{(n)}(t), \ldots\right) \in \ell_{0}^{\downarrow}
$$

the sequence of component sizes of $F_{t}^{(n)}$, arranged in decreasing order.

Then $\mathbf{M}^{(n)}(t), t \geq 0$ is a Markov process - let us call it here the frozen percolation component process on $n$ vertices with lightning rate $\lambda(n)$, or briefly $\operatorname{FP}(n, \lambda(n))$. In fact, up to time-change, $\mathbf{M}^{(n)}(t), t \geq 0$ evolves according to the rules (1.2) of MCLD.

Remark 1.6. We note that $\operatorname{FP}(n, \lambda(n))$ is a simplification of the mean field forest fire model [19], the definition of which agrees with Definition 1.5 above, with the only difference that in 
the forest fire model we only delete the edges of the connected components that are destroyed by fire, that is, a destroyed component of size $k$ is immediately replaced by $k$ singletons. The mean field forest fire model behaves very similarly to the mean field frozen percolation model (e.g., the self-organized critical behaviour of the two models are quite similar, see also Remark 1.10(iii) below), however the mathematics of the mean field frozen percolation model is simpler than that of the mean field forest fire model, for example, the solution of the system of differential equations that appears in [18], Theorem 1.2, is fairly explicit, while the system of differential equations that appears in [19], Theorem 2, currently does not have an explicit solution.

Remark 1.7. One studies the asymptotic behaviour of the component size structure of $\operatorname{FP}(n, \lambda(n))$ when $1 \ll n$ and $1 / n \ll \lambda(n) \ll 1$. Definition 1.5 above is slightly different from the one proposed in [5], Section 5.5, and studied in [17] where connected components are frozen when their size exceeds a threshold $\omega(n)$ satisfying $1 \ll \omega(n) \ll n$. The results [17], Theorem 1.1, and [18], Theorem 1.2, are very similar: indeed, if one is interested in small connected component densities then the two models produce exactly the same (self-organized critical) behaviour. However, if one is interested in the scaling limit of big component dynamics, the exact deletion mechanism does crucially enter the picture.

We are interested in identifying the scaling limit of $\operatorname{FP}(n, \lambda(n))$ as $n \rightarrow \infty$. In order to describe the kind of result we are after, let us recall that the large components of the dynamical Erdős-Rényi random graph process in the critical window $\mathcal{G}\left(n, \frac{1+t n^{-1 / 3}}{n}\right), t \in \mathbb{R}$, scaled by $n^{2 / 3}$, converge in law to the standard multiplicative coalescent process $(\mathcal{M}(t), t \in \mathbb{R})$, see [3], Section 4.3.

Remark 1.8. The family of multiplicative coalescent processes defined for all $t \in \mathbb{R}$ (i.e., the eternal MC processes) are characterized in [4]. The class of inhomogeneous random graph models whose scaling limit is the standard MC is explored in [6,8] (see also references therein). The scaling limits of other classes of inhomogeneous random graph models are related to nonstandard eternal MC processes, see [4,9]. The continuum scaling limit of the metric structure of critical random graphs is studied in $[1,7]$ (see also references therein).

The next result gives a scaling limit for the frozen percolation process started from a critical Erdős-Rényi graph.

Proposition 1.9. Fix $u \in \mathbb{R}$ and let $F_{0}^{(n)}$ be an Erdôs-Rényi graph $\mathcal{G}(n, p)$ with edge probability $p=\frac{1+u n^{-1 / 3}}{n}$. Let $\lambda>0$ and let $\mathbf{M}^{(n)}(t), t \geq 0$ be the $\operatorname{FP}\left(n, \lambda n^{-1 / 3}\right)$ process with initial state $F_{0}^{(n)}$. Define $\mathbf{m}^{(n)}(t), t \geq 0$ by

$$
\mathbf{m}^{(n)}(t):=\left(n^{-2 / 3} M_{1}^{(n)}\left(n^{-1 / 3} t\right), n^{-2 / 3} M_{2}^{(n)}\left(n^{-1 / 3} t\right), \ldots\right) .
$$

Then as $n \rightarrow \infty$ the finite dimensional marginals of the sequence of $\ell_{2}^{\downarrow}$-valued processes $\mathbf{m}^{(n)}(t), t \geq 0$ converge in law to the finite dimensional marginals of the MCLD $(\lambda)$ process $(\mathbf{m}(t), t \geq 0)$ started from an initial state with distribution $\mathbf{m}(0) \sim \mathcal{M}(u)$ (i.e., the state of the 
standard multiplicative coalescent process at time $u$ ), i.e., for every $k \in \mathbb{N}$ and $0 \leq t_{1}<t_{2}<$ $\cdots<t_{k}$ we have

$$
\left(\mathbf{m}^{(n)}\left(t_{1}\right), \mathbf{m}^{(n)}\left(t_{2}\right), \ldots, \mathbf{m}^{(n)}\left(t_{k}\right)\right) \stackrel{\mathrm{d}}{\longrightarrow}\left(\mathbf{m}\left(t_{1}\right), \mathbf{m}\left(t_{2}\right), \ldots, \mathbf{m}\left(t_{k}\right)\right), \quad n \rightarrow \infty .
$$

The proof of Proposition 1.9 follows as an application of Theorem 1.2 (for details of the proof, we refer to [15], Proposition 6.10).

Remark 1.10. (i) Loosely speaking, if $(\mathbf{m}(t), t \geq 0)$ is the $\operatorname{MCLD}(\lambda)$ process started from an initial state with distribution $\mathbf{m}(0) \sim \mathcal{M}(u)$ (this is the limit object that appears in Proposition 1.9), then we have $\mathbf{m}(t) \sim \mathcal{M}(u+t-\Phi(t))$, where $\Phi(t)$ denotes the sum of the sizes of the components deleted up to time $t$ (see [15], Proposition 6.7(ii), for a precise formulation of this property). In fact, in [15], Proposition 6.7, we give a representation of $(\mathbf{m}(t), t \geq 0)$ on the probability space of a standard Brownian motion using what we call the "rigid" representation of $\operatorname{MCLD}(\lambda)$. We note that Theorem 1.2 is also crucially used when we extend our rigid representation results from $\ell_{0}^{\downarrow}$ to $\ell_{2}^{\downarrow}$ in [15], Section 5 .

(ii) In [16], we describe the possible scaling limits that can arise from a $\operatorname{FP}\left(n, \lambda n^{-1 / 3}\right)$ process started from an empty graph. The possible limit objects are eternal $\operatorname{MCLD}(\lambda)$ processes (i.e., they are defined for any $t \in \mathbb{R}$ ). The "arrival at the critical window" gives rise to a nonstationary $\operatorname{MCLD}(\lambda)$ scaling limit, while the scaling limit in the "self-organized critical" regime is a stationary $\operatorname{MCLD}(\lambda)$ (see also [15], Remark 6.8).

(iii) We conjecture that the scaling limit of the coagulation-fragmentation dynamics of big components of the mean field forest fire model (cf. Remark 1.6 above) with lightning rate $\lambda n^{-1 / 3}$ is also an $\operatorname{MCLD}(\lambda)$ process.

\section{Notation and basic results}

The aim of this section is to collect some basic results about the multiplicative coalescent from [3] and [14]. In some cases, we will augment these results to fit our purposes or present them using different notation.

We define

$$
\ell_{2}^{+}=\left\{x=\left(x_{1}, x_{2}, \ldots\right): \forall i x_{i} \geq 0, \sum_{i \geq 1} x_{i}^{2}<+\infty\right\}
$$

We have $\ell_{2}^{\downarrow} \subseteq \ell_{2}^{+}$. Define the mapping

$$
\text { ord : } \quad \ell_{2}^{+} \rightarrow \ell_{2}^{\downarrow}
$$

by letting $\operatorname{ord}(\underline{x})$ be the decreasing rearrangement of $\underline{x} \in \ell_{2}^{+}$.

Definition 2.1. If $\underline{m} \in \ell_{2}^{\downarrow}$ and $G$ is a graph with vertex set $V \subseteq \mathbb{N}_{+}$, denote by $\operatorname{ord}(\underline{m}, G)$ the ordered sequence of the weights of the connected components of $G$. More precisely, if $\overline{\mathcal{C}_{1}}, \mathcal{C}_{2}, \ldots$ 
is the sequence of the vertex sets of the connected components of $G$, we define

$$
\underline{x}_{G}=\left(\sum_{i \in \mathcal{C}_{1}} m_{i}, \sum_{i \in \mathcal{C}_{2}} m_{i}, \ldots\right) \text { and } \operatorname{ord}(\underline{m}, G) \stackrel{(2.1)}{=} \operatorname{ord}\left(\underline{x}_{G}\right),
$$

assuming that $\underline{x}_{G} \in \ell_{2}^{+}$. We also denote

$$
S_{2}^{G}=\sum_{k=1}^{\infty}\left(\sum_{j \in \mathcal{C}_{k}} m_{j}\right)^{2}=\left\|x_{G}\right\|_{2}^{2}=\left\|\operatorname{ord}\left(\underline{x}_{G}\right)\right\|_{2}^{2} .
$$

Let us now state an elementary yet useful result which involves the metric $\mathrm{d}(\cdot, \cdot)$ defined in (1.1).

Lemma 2.2. If $\underline{m} \in \ell_{2}^{\downarrow}$ and $G, G^{\prime}$ are graphs with vertex sets $V, V^{\prime} \subseteq \mathbb{N}_{+}$such that $V \subseteq V^{\prime}$, $G \subseteq G^{\prime}$ and $\operatorname{ord}(\underline{m}, G) \in \ell_{2}^{\downarrow}$, then we have

$$
\mathrm{d}\left(\operatorname{ord}(\underline{m}, G), \operatorname{ord}\left(\underline{m}, G^{\prime}\right)\right) \leq \sqrt{\left\|\operatorname{ord}\left(\underline{m}, G^{\prime}\right)\right\|_{2}^{2}-\|\operatorname{ord}(\underline{m}, G)\|_{2}^{2}} .
$$

Proof. This is a special case of [3], Lemma 17.

Let us recall the graphical construction used in [3], Section 1.5, to define the multiplicative coalescent process.

Definition 2.3. Let $\left(\xi_{i, j}\right)_{1 \leq i<j<\infty}$ denote independent random variables with EXP(1) distribution. Given $\underline{x} \in \ell_{2}^{+}$let us define the simple graph $G_{t}$ with vertex set $\mathbb{N}_{+}$and an edge between $i$ and $j$ if and only if $\xi_{i, j} \leq t x_{i} x_{j}$. For $i, j \in \mathbb{N}_{+}$we denote by $i \stackrel{G_{t}}{\longleftrightarrow} j$ the event that $i$ and $j$ are connected by a simple path in the graph $G_{t}$.

Given $G_{t}$ we define the connected components $\left(\mathcal{C}_{k}(t)\right)_{k=1}^{\infty}$ of $G_{t}$ by

$$
i_{k}=\min \left\{\mathbb{N}_{+} \backslash \bigcup_{l=1}^{k-1} \mathcal{C}_{l}(t)\right\}, \quad \mathcal{C}_{k}(t)=\left\{i \in \mathbb{N}_{+}: i \stackrel{G_{t}}{\longleftrightarrow} i_{k}\right\}, \quad k \geq 1 .
$$

Note that we have

$$
S_{2}^{G_{t}} \stackrel{(2.3)}{=} S_{2}^{G_{0}}+\sum_{i \neq j} x_{i} x_{j} \mathbb{1}\left[i \stackrel{G_{t}}{\longleftrightarrow} j\right]
$$

and $S_{2}^{G_{0}}=\sum_{i=1}^{\infty} x_{i}^{2}<+\infty$ if $\underline{x} \in \ell_{2}^{+}$.

The statement of the next lemma follows from [3], Proposition 5, and shows that Definitions 2.1 and 2.3 give rise to a graphical representation of the $\ell_{2}^{\downarrow}$-valued multiplicative coalescent process with initial state $\underline{m} \in \ell_{2}^{\downarrow}$ in the form $\operatorname{ord}\left(\underline{m}, G_{t}\right), t \geq 0$. 
Lemma 2.4. For any $t \geq 0$ and $\underline{x} \in \ell_{2}^{+}$we have

$$
\mathbf{P}\left(S_{2}^{G_{t}}<+\infty\right)=1
$$

In particular, for any $t \in \mathbb{R}_{+}$the weights of the connected components of $G_{t}$ are almost surely finite:

$$
\mathbf{P}\left(\forall k \in \mathbb{N}_{+}: \sum_{i \in \mathcal{C}_{k}(t)} x_{i}<+\infty\right)=1
$$

The next lemma is an extended version of [14], (2.2).

Lemma 2.5. For any $\underline{x} \in \ell_{2}^{+}$and $i, j \in \mathbb{N}_{+}$and $t<\frac{1}{S_{2}^{G_{0}}}$ we have

$$
\mathbf{P}\left(i \stackrel{G_{t}}{\longleftrightarrow} j\right) \leq \frac{x_{i} \cdot x_{j} \cdot t}{1-t \cdot S_{2}^{G_{0}}} .
$$

\section{Proof.}

$$
\begin{aligned}
\mathbf{P} & \left(i \stackrel{G_{t}}{\longleftrightarrow} j\right) \\
& \leq \sum_{k=1}^{\infty} \mathbf{P}\left(\begin{array}{c}
\exists i_{0}, \ldots, i_{k} \in \mathbb{N}_{+}: i_{0}=i, i_{k}=j \text { and } \\
\left(i_{0}, i_{1}, \ldots, i_{k-1}, i_{k}\right) \text { is a simple path in } G_{t}
\end{array}\right) \\
& \leq \sum_{k=1}^{\infty} \sum_{\left(i_{1}, \ldots, i_{k-1}\right) \in \mathbb{N}_{+}^{k-1}} \prod_{l=1}^{k}\left(1-\exp \left(-x_{i_{l-1}} x_{i_{l}} t\right)\right) \\
& \leq \sum_{k=1}^{\infty} \sum_{\left(i_{1}, \ldots, i_{k-1}\right) \in \mathbb{N}_{+}^{k-1}} \prod_{l=1}^{k} x_{i_{l-1}} x_{i_{l}} t=x_{i} x_{j} t \cdot \sum_{k=1}^{\infty} \sum_{\left(i_{1}, \ldots, i_{k-1}\right) \in \mathbb{N}_{+}^{k-1}} \prod_{l=1}^{k-1} x_{i_{l}}^{2} t \\
& =x_{i} x_{j} t \cdot \sum_{k=1}^{\infty}\left(t \cdot S_{2}^{G_{0}}\right)^{k-1}=\frac{x_{i} \cdot x_{j} \cdot t}{1-t \cdot S_{2}^{G_{0}}} .
\end{aligned}
$$

Corollary 2.6. For any $\underline{x} \in \ell_{2}^{+}, t \geq 0$ and $i, j \in \mathbb{N}_{+}$, if

$$
S_{2}^{G_{0}} \leq \frac{1}{2 t}
$$

holds then we have

$$
\mathbf{E}\left(S_{2}^{G_{t}}\right) \leq 2 S_{2}^{G_{0}}
$$


Proof. Using (2.5), (2.8) and (2.10), we obtain

$$
\mathbf{E}\left(S_{2}^{G_{t}}\right) \leq S_{2}^{G_{0}}+2 t \sum_{i \neq j} x_{i}^{2} x_{j}^{2} \leq S_{2}^{G_{0}}+2 t \cdot\left(S_{2}^{G_{0}}\right)^{2} \stackrel{(2.10)}{\leq} 2 S_{2}^{G_{0}} .
$$

The next lemma is based on [3], Lemma 23, and [14], (2.5). It will be used in Section 4 to show that the truncated process is close to the original process if the truncation threshold is chosen big enough.

Lemma 2.7. Let $\underline{x}, \underline{y} \in \ell_{2}^{+}$and $t \geq 0$. Denote the index set of $\underline{x}$ by I and the index set of $\underline{y}$ by $J$. Denote by

$$
a=\|\underline{x}\|_{2}^{2}<+\infty \quad \text { and } \quad b=\|\underline{y}\|_{2}^{2}<+\infty .
$$

Consider the bipartite random graph $B_{t}$ with vertex set $I \cup J$, where $i \in I$ and $j \in J$ are connected with probability $1-\exp \left(-t x_{i} y_{j}\right)$. Then we have

$$
|I|<+\infty \quad \Longrightarrow \quad \mathbf{E}\left(S_{2}^{B_{t}}\right)<+\infty \text {. }
$$

Moreover, if

$$
t^{2} a b \leq \frac{1}{2}
$$

holds then we have

$$
\mathbf{E}\left(S_{2}^{B_{t}}\right)-a \leq 2 b \cdot(1+t a)^{2}
$$

Proof. First note that, similarly to (2.5), we have

$$
\begin{aligned}
\mathbf{E}\left(S_{2}^{B_{t}}\right)= & a+b+\sum_{i_{1} \neq i_{2} \in I} x_{i_{1}} x_{i_{2}} \mathbf{P}\left(i_{1} \stackrel{B_{t}}{\longleftrightarrow} i_{2}\right) \\
& +\sum_{j_{1} \neq j_{2} \in J} y_{j_{1}} y_{j_{2}} \mathbf{P}\left(j_{1} \stackrel{B_{t}}{\longleftrightarrow} j_{2}\right)+2 \sum_{i \in I, j \in J} x_{i} y_{j} \mathbf{P}\left(i \stackrel{B_{t}}{\longleftrightarrow} j\right) .
\end{aligned}
$$

Now note that the number of visits to $I$ of a simple path in $B_{t}$ is at most $|I|$. Using this idea and a calculation similar to (2.9), we obtain the inequalities

$$
\begin{gathered}
\mathbf{P}\left(i_{1} \stackrel{B_{t}}{\longleftrightarrow} i_{2}\right) \leq\left(x_{i_{1}} x_{i_{2}} \cdot b \cdot t^{2}\right) \cdot \sum_{k=1}^{|I|}\left(t^{2} a b\right)^{k-1}, \quad i_{1} \neq i_{2}, i_{1}, i_{2} \in I, \\
\mathbf{P}\left(j_{1} \stackrel{B_{t}}{\longleftrightarrow} j_{2}\right) \leq\left(y_{j_{1}} y_{j_{2}} \cdot a \cdot t^{2}\right) \cdot \sum_{k=1}^{|I|}\left(t^{2} a b\right)^{k-1}, \quad j_{1} \neq j_{2}, j_{1}, j_{2} \in J, \\
\mathbf{P}\left(i \stackrel{B_{t}}{\longleftrightarrow} j\right) \leq\left(x_{i} y_{j} t\right) \cdot \sum_{k=1}^{|I|}\left(t^{2} a b\right)^{k-1}, \quad i \in I, j \in J .
\end{gathered}
$$


Combining these inequalities with (2.16) we obtain (2.13) as well as

$$
\begin{array}{r}
\mathbf{E}\left(S_{2}^{B_{t}}\right)-a \stackrel{(2.14)}{\leq} b+2\left(a^{2} \cdot b \cdot t^{2}+b^{2} \cdot a \cdot t^{2}+2 a \cdot b \cdot t\right) \\
\stackrel{(2.14)}{\leq} b \cdot\left(1+2 a^{2} t^{2}+1+4 a t\right)=2 b(1+a t)^{2} .
\end{array}
$$

This completes the proof of (2.15).

Lemma 2.8. With probability 1 , the function $t \mapsto \operatorname{ord}\left(\underline{m}, G_{t}\right)$ (see (2.2)) is càdlàg with respect to the $\mathrm{d}(\cdot, \cdot)$-metric (defined in (1.1)).

Proof. Let us fix some $T \geq 0$. Denote by $A$ the event

$$
A=\left\{S_{2}^{G_{T}}<+\infty\right\} \cap\left\{\begin{array}{c}
\text { for any } i, j \in \mathbb{N} \text { the number of simple } \\
\text { paths connecting } i \text { and } j \text { in } G_{T} \text { is finite }
\end{array}\right\} .
$$

By Lemma 2.4 the event $A$ almost surely holds. Assuming that $A$ holds, we will show that $t \mapsto \operatorname{ord}\left(\underline{m}, G_{t}\right)$ is càdlàg on $[0, T)$.

Since $\bar{G}_{s} \subseteq G_{t}$ if $s \leq t$, we can apply Lemma 2.2 in order to reduce our task to showing that the function $t \mapsto S_{2}^{G_{t}}$ is càdlàg on $[0, T)$. If $A$ holds, then for any $i, j \in \mathbb{N}$ the function $t \mapsto$ $\mathbb{1}\left[i \stackrel{G_{t}}{\longleftrightarrow} j\right]$ is càdlàg on $[0, T)$. Using this fact, $(2.5)$ and the dominated convergence theorem, we obtain that indeed $t \mapsto S_{2}^{G_{t}}$ is also càdlàg on $[0, T)$.

\section{Graphical construction of $\operatorname{MCLD}(\lambda)$}

Recall the informal definition of the $\operatorname{MCLD}(\lambda)$ process $\mathbf{m}_{t}$ from (1.2). We now give a graphical construction of the process $\mathbf{m}_{t}$ with initial state $\underline{m} \in \ell_{2}^{\downarrow}$ and deletion rate $\lambda$. Let

$$
\begin{aligned}
\left(\xi_{i, j}\right)_{1 \leq i<j<\infty} & \text { be random variables with } \operatorname{EXP}(1) \text { distribution, } \\
\left(\lambda_{i}\right)_{1 \leq i<\infty} & \text { be random variables with } \operatorname{EXP}(\lambda) \text { distribution, }
\end{aligned}
$$

and let us also assume that all of these random variables are independent.

The heuristic description of our graphical construction is as follows: we increase $t$ continuously and if the event $\xi_{i, j}=t m_{i} m_{j}$ occurs for some $1 \leq i<j<\infty$, we merge the components of the vertices $i$ and $j$, moreover, if $\lambda_{i}=t m_{i}$ for some $i \in \mathbb{N}_{+}$, then we say that a lightning strikes vertex $i$ and delete the connected component of vertex $i$. Since the total rate of merger and deletion events is infinite if $\sum_{i} m_{i}=+\infty$, we need to be careful with the above heuristic definition if we want to make it precise: we will now provide the graphical construction.

In Definition 2.3, we defined the simple graph $G_{t}$ with vertex set $\mathbb{N}_{+}$.

We will define for any $t \in \mathbb{R}_{+}$

$$
\begin{aligned}
& \text { the set of intact vertices } \mathcal{V}_{t} \subseteq \mathbb{N}_{+} \text {and } \\
& \text { the set of burnt vertices } \mathbb{N}_{+} \backslash \mathcal{V}_{t}
\end{aligned}
$$


The graph $H_{t}$ will denote the subgraph of $G_{t}$ spanned by $\mathcal{V}_{t}$ and $\mathbf{m}_{t}$ will denote the ordered sequence of component weights of $H_{t}$.

Recall that we enumerated the connected components $\mathcal{C}_{k}(t), k \in \mathbb{N}_{+}$of $G_{t}$ in (2.4). By the properties of exponential random variables, (2.7) and the independence of the exponential random variables $\left(\xi_{i, j}\right)_{1 \leq i<j<\infty}$ and $\left(\lambda_{i}\right)_{i=1}^{\infty}$, we see that for every $t \geq 0$

$$
\mathbf{P}\left(\forall k \in \mathbb{N}_{+}: \sum_{i \in \mathcal{C}_{k}(t)} \mathbb{1}\left[\lambda_{i} \leq t m_{i}\right]<+\infty\right)=1 .
$$

This implies that for every $t \geq 0$ and $k \in \mathbb{N}_{+}$, there exists an almost surely finite $\mathbb{N}$-valued random variable $N$ (the number of lightnings that hit the component $\mathcal{C}_{k}(t)$ by time $t$ ), indices $i_{1}, \ldots, i_{N} \subseteq \mathcal{C}_{k}(t)$ (the vertices that are hit by lightning) and times $0<t_{1}<\cdots<t_{N} \leq t$ (the ordered sequence of the times of the lightnings) such that

$$
\left\{i \in \mathcal{C}_{k}(t): \lambda_{i} \leq t m_{i}\right\}=\left\{i_{1}, \ldots, i_{N}\right\} \quad \text { and } \quad \forall 1 \leq l \leq N: t_{l}=\frac{\lambda_{i_{l}}}{m_{i_{l}}}
$$

We now define the set of intact vertices $\mathcal{V}_{t} \subseteq \mathbb{N}_{+}$by constructing $\mathcal{V}_{t} \cap \mathcal{C}_{k}(t)$ for every $k \in \mathbb{N}_{+}$. Let us fix $k \in \mathbb{N}_{+}$. We recursively define $\mathcal{V}_{t_{l}} \cap \mathcal{C}_{k}(t)$ for each $1 \leq l \leq N$ in the following way.

(i) At $t_{0}=0$ we have $\mathcal{V}_{t_{0}} \cap \mathcal{C}_{k}(t)=\mathcal{C}_{k}(t)$.

(ii) Assume that we have already constructed $\mathcal{V}_{t_{l-1}} \cap \mathcal{C}_{k}(t)$ for some $1 \leq l \leq N$. We define $\mathcal{V}_{t_{l}} \cap \mathcal{C}_{k}(t)$ by deleting the connected component of $i_{l}$ in the restriction of the graph $G_{t_{l}}$ to the vertex set $\mathcal{V}_{t_{l-1}} \cap \mathcal{C}_{k}(t)$.

(iii) With this recursion we define $\mathcal{V}_{t_{N}} \cap \mathcal{C}_{k}(t)$. Since there are no lightnings hitting $\mathcal{C}_{k}(t)$ between $t_{N}$ and $t$, let $\mathcal{V}_{t} \cap \mathcal{C}_{k}(t)=\mathcal{V}_{t_{N}} \cap \mathcal{C}_{k}(t)$.

Since $\mathcal{C}_{k}(t), k \in \mathbb{N}_{+}$is a partition of $\mathbb{N}_{+}$, we define

$$
\mathcal{V}_{t}=\bigcup_{k \geq 1}\left(\mathcal{V}_{t} \cap \mathcal{C}_{k}(t)\right) \quad \text { and }
$$

$H_{t}$ to be the subgraph of $G_{t}$ spanned by $\mathcal{V}_{t}$.

Recalling Definition 2.1 we let

$$
\mathbf{m}_{t}=\operatorname{ord}\left(\underline{m}, H_{t}\right) \text {. }
$$

Lemma 3.1. For any $\underline{m} \in \ell_{2}^{\downarrow}$ the graphical construction (3.5) of the process $\mathbf{m}_{t}$ gives an $\operatorname{MCLD}(\lambda)$ process with initial condition $\underline{m}$, i.e., an $\ell_{2}^{\downarrow}$-valued Markov process whose dynamics satisfy the informal definition given in (1.2).

Proof. $\mathbf{m}_{t}$ is a random element of $\ell_{2}^{\downarrow}$, because we have

$$
\left\|\mathbf{m}_{t}\right\|_{2}^{2}=S_{2}^{H_{t}} \leq S_{2}^{G_{t}} \stackrel{(2.6)}{<}+\infty .
$$


The fact that $\mathbf{m}_{t}$ is a Markov process with the prescribed transition rates follows from the memoryless property and independence of the random variables $\left(\xi_{i, j}\right)_{1 \leq i<j<\infty}$ and $\left(\lambda_{i}\right)_{i=1}^{\infty}$. We omit further details.

Proof of Proposition 1.1. We will show that with probability 1 , the function $t \mapsto \operatorname{ord}\left(\underline{m}, H_{t}\right)$ is càdlàg with respect to the $\mathrm{d}(\cdot, \cdot)$-metric, see $(1.1)$.

Let us fix some $T \geq 0$. We know that the event $A$ defined in (2.17) almost surely holds. Denote by $B$ the event that every connected component of $G_{T}$ is exposed to only finitely many lightning strikes on $[0, T]$. By (3.3), the event $B$ occurs almost surely. Assuming that $A \cap B$ holds, we will show that $t \mapsto \operatorname{ord}\left(\underline{m}, H_{t}\right)$ is càdlàg on $[0, T)$. For any $t \geq 0$, define

- $\hat{H}_{t+\Delta t}$ to be the subgraph of $G_{t}$ spanned by $\mathcal{V}_{t+\Delta t}$,

- $\check{H}_{t+\Delta t}$ to be the subgraph of $G_{t+\Delta t}$ spanned by $\mathcal{V}_{t}$.

Recalling (3.4) and the inclusions $G_{t} \subseteq G_{t+\Delta t}$ and $\mathcal{V}_{t+\Delta t} \subseteq \mathcal{V}_{t}$ we see that

$$
\hat{H}_{t+\Delta t} \subseteq H_{t} \subseteq \check{H}_{t+\Delta t} \quad \text { and } \quad \hat{H}_{t+\Delta t} \subseteq H_{t+\Delta t} \subseteq \check{H}_{t+\Delta t}
$$

so we can apply Lemma 2.2 and the triangle inequality in order to reduce our task of proving right-continuity of $t \mapsto \operatorname{ord}\left(\underline{m}, H_{t}\right)$ at $t$ to showing that

$$
\text { (a) } \quad \lim _{\Delta t \rightarrow 0_{+}} S_{2}^{\check{H}_{t+\Delta t}}-S_{2}^{H_{t}}=0, \quad \text { (b) } \quad \lim _{\Delta t \rightarrow 0_{+}} S_{2}^{H_{t}}-S_{2}^{\hat{H}_{t+\Delta t}}=0 \text {. }
$$

Now (a) follows from the fact that the graphical representation of the multiplicative coalescent possesses the càdlàg property (see Lemma 2.8 ).

In order to show (b) we observe that on the event $B$, for every connected component $\mathcal{C}$ of $G_{T}$, we have

$$
\lim _{\Delta t \rightarrow 0} \mathbb{1}\left[\exists i \in \mathcal{C}: t m_{i}<\lambda_{i} \leq(t+\Delta t) m_{i}\right]=0 .
$$

Given this observation, we see that for every connected component $\mathcal{C}$ of $H_{t}$ we have $\lim _{\Delta t \rightarrow 0} \mathbb{1}\left[\mathcal{C} \subseteq \mathcal{V}_{t+\Delta t}\right]=1$. Using this fact, $S_{2}^{H_{t}}<\infty$ and the dominated convergence theorem, we obtain (b).

The proof of the existence of left limits is similar and we omit it.

\section{Feller property of $\operatorname{MCLD}(\lambda)$}

Definition 4.1. The graphical construction of Section 3 gives a joint realization of all of the $\operatorname{MCLD}(\lambda)$ processes with different initial conditions by using the same collection of random variables $\left(\xi_{i, j}\right)_{1 \leq i<j<\infty}$ and $\left(\lambda_{i}\right)_{1 \leq i<\infty}$ (see (3.1)). We call this coupling the $(\xi, \lambda)$-coupling.

Theorem 4.2. Let $\underline{m}^{(n)}, n \in \mathbb{N}$ be a convergent sequence of elements of $\ell_{2}^{\downarrow}$ and let $\underline{m}^{(\infty)}$ denote their limit, i.e., $\lim _{n \rightarrow \infty} \mathrm{d}\left(\underline{m}^{(n)}, \underline{m}^{(\infty)}\right)=0$. For any $t \in \mathbb{R}_{+}$and $n \in \mathbb{N}_{+} \cup\{\infty\}$, denote by $\mathbf{m}_{t}^{(n)}$ 
the $\operatorname{MCLD}(\lambda)$ process with initial condition $\underline{m}^{(n)}$ at time $t$. Under the $(\xi, \lambda)$-coupling, we have

$$
\mathrm{d}\left(\mathbf{m}_{t}^{(n)}, \mathbf{m}_{t}^{(\infty)}\right) \stackrel{p}{\longrightarrow} 0, \quad n \rightarrow \infty .
$$

Theorem 4.2 implies that the $\operatorname{MCLD}(\lambda)$ Markov process indeed possesses the Feller property, that is, Theorem 1.2 holds.

We want to prove Theorem 4.2 using truncation, because (4.1) trivially holds for the truncated process. However, we cannot directly apply Lemma 2.2 to compare the original with the truncated process, because we cannot upper bound the state of the truncated process at time $t$ by the state of the original process at time $t$ (cf. Remark 1.3).

In Section 4.1, we overcome this problem by introducing two auxiliary objects that upper/lower bound both the original and the truncated object, but yet these auxiliary objects can be shown to be close to each other if we only throw away a small part of the original when we truncate.

In Section 4.2, we prove Theorem 4.2 using the results of Section 4.1 and variant of the $\varepsilon / 3$ argument.

\subsection{Bounding the effect of truncation}

In this subsection, we will fix $t \geq 0$ as well as an initial state $\underline{m} \in \ell_{2}^{\downarrow}$, and omit the dependence of random variables on $t$ and $\underline{m}$. We also fix a truncation threshold $m \in \mathbb{N}$.

Definition 4.3. Recall Definition 2.3. Denote by $G, G^{m \downarrow}$ and $G^{m \uparrow}$ the graphs with adjacency matrix $\mathbb{1}\left[\xi_{i, j} \leq t m_{i} m_{j}\right]$ on the vertex set $\mathbb{N}_{+},\{1, \ldots, m\}$, and $\{m+1, m+2, \ldots\}$, respectively.

Let $\underline{m}^{(m)}$ denote the vector $\underline{m}$ truncated at index $m$ :

$$
\underline{m}^{(m)}=\left(m_{1}, \ldots, m_{m}, 0,0, \ldots\right), \quad \text { where } \underline{m}=\left(m_{1}, m_{2}, \ldots\right) .
$$

Let $\mathbf{m}$ (resp. $\left.\mathbf{m}^{(m)}\right)$ denote the state at time $t$ of the realization under the $(\xi, \lambda)$-coupling of the $\operatorname{MCLD}(\lambda)$ process with initial state $\underline{m}$ (resp. $\left.\underline{m}^{(m)}\right)$.

Denote by $\mathcal{V}$ and $\mathcal{V}^{(m)}$ the corresponding sets of intact vertices, see (3.4).

Denote by $H$ and $H^{(m)}$ the subgraphs of $G$ spanned by $\mathcal{V}$ and $\mathcal{V}^{(m)}$.

In order to compare $\mathbf{m}$ with $\mathbf{m}^{(m)}$, we need the following result.

Lemma 4.4. If $\hat{G}^{(m)}$ and $\check{G}^{(m)}$ are random graphs with vertex sets

$$
V\left(\hat{G}^{(m)}\right), V\left(\check{G}^{(m)}\right) \subseteq \mathbb{N}_{+}
$$

and under the $(\xi, \lambda)$-coupling we have

$$
\hat{G}^{(m)} \subseteq H^{(m)} \subseteq \check{G}^{(m)}, \quad \hat{G}^{(m)} \subseteq H \subseteq \check{G}^{(m)}
$$

then almost surely we have

$$
\mathrm{d}\left(\mathbf{m}, \mathbf{m}^{(m)}\right) \leq 3 \cdot \sqrt{S_{2}^{\check{G}^{(m)}}-S_{2}^{\hat{G}^{(m)}}}
$$


Proof. First note that it follows from (4.2) that

$$
S_{2}^{\hat{G}^{(m)}} \leq S_{2}^{H^{(m)}} \leq S_{2}^{\check{G}^{(m)}}, \quad S_{2}^{\hat{G}^{(m)}} \leq S_{2}^{H} \leq S_{2}^{\check{G}^{(m)}}
$$

Thus, we have

$$
\begin{aligned}
\mathrm{d}\left(\mathbf{m}, \mathbf{m}^{(m)}\right) \stackrel{(2.2)}{\leq} & \mathrm{d}\left(\mathbf{m}, \operatorname{ord}\left(\underline{m}, \check{G}^{(m)}\right)\right) \\
& +\mathrm{d}\left(\operatorname{ord}\left(\underline{m}, \check{G}^{(m)}\right), \operatorname{ord}\left(\underline{m}^{(m)}, \hat{G}^{(m)}\right)\right)+\mathrm{d}\left(\operatorname{ord}\left(\underline{m}^{(m)}, \hat{G}^{(m)}\right), \mathbf{m}^{(m)}\right) \\
& \stackrel{(*)}{\leq} \sqrt{S_{2}^{\check{G}^{(m)}}-S_{2}^{H}}+\sqrt{S_{2}^{\check{G}^{(m)}}-S_{2}^{\hat{G}^{(m)}}}+\sqrt{S_{2}^{H^{(m)}}-S_{2}^{\hat{G}^{(m)}}} \stackrel{(4.4)}{\leq} 3 \cdot \sqrt{S_{2}^{\check{G}^{(m)}}-S_{2}^{\hat{G}^{(m)}}},
\end{aligned}
$$

where $(*)$ follows from (3.5), the inclusions (4.2) and Lemma 2.2.

In Definition 4.8 below, we will construct auxiliary graphs $\hat{G}^{(m)}$ and $\check{G}^{(m)}$ in such a way that (4.2) holds. Recall Definition 4.3. Note that $H^{(m)}$ is the subgraph of $G^{m \downarrow}$ spanned by the vertex set $\mathcal{V}^{(m)}$. In particular, every connected component of $H^{(m)}$ is a subset of a connected component of $G^{m \downarrow}$.

The next definition only involves the random variables $\left(\xi_{i, j}\right)_{1 \leq i<j<\infty}$ (i.e., we don't have to look at $\left.\left(\lambda_{i}\right)_{i=1}^{\infty}\right)$.

Definition 4.5. Given $G^{m \downarrow}$ and $G^{m \uparrow}$, denote the connected components of $G^{m \downarrow}$ by $\mathcal{C}_{k}^{m \downarrow}, k \in K$ and the connected components of $G^{m \uparrow}$ by $\mathcal{C}_{l}^{m \uparrow}, l \in L$.

Let us define an auxiliary bipartite multigraph $\mathcal{B}$ with vertex set $K \cup L$. Declare $k \in K$ and $l \in L$ connected in $\mathcal{B}$ if $\mathcal{C}_{k}^{m \downarrow}$ is connected to $\mathcal{C}_{l}^{m \uparrow}$ in $G$. We allow parallel edges to be present in $\mathcal{B}$ : if $\mathcal{C}_{k}^{m \downarrow}$ is connected to $\mathcal{C}_{l}^{m \uparrow}$ by more than one edge in $G$, then we put an equal number of parallel edges between $k \in K$ and $l \in L$ in $\mathcal{B}$.

Now we define a subset $K^{*} \subseteq K$ indexing "bad" components of $G^{m \downarrow}$. This definition involves the random variables $\left(\xi_{i, j}\right)_{1 \leq i<j<\infty}$ as well as $\left(\lambda_{i}\right)_{i=1}^{\infty}$. The components indexed by $k \in K \backslash K^{*}$ are "good". The key property of good components will be stated in Lemma 4.7 below.

Definition 4.6. Recall the definition of $\mathcal{B}$ from Definition 4.5.

(i) An edge-simple path in $\mathcal{B}$ is a path with no repeated edges.

(ii) We say that $k \in K$ (resp. $l \in L$ ) is intact if no lightning hit any vertex of $\mathcal{C}_{k}^{m \downarrow}$ (resp. $\mathcal{C}_{l}^{m \uparrow}$ ) before time $t$. If a vertex of $\mathcal{B}$ is not intact, then we say that it is damaged.

(iii) We say that $k \in K^{*}$ if $k \in K$ and there is a edge-simple path in $\mathcal{B}$ which consists of at least one edge and connects $k$ to a damaged vertex of $\mathcal{B}$.

For an illustration of Definition 4.6, see Figure 1.

Lemma 4.7. Recalling Definition 4.3, we have

$$
\forall k \in K \backslash K^{*}: \mathcal{C}_{k}^{m \downarrow} \cap \mathcal{V}^{(m)}=\mathcal{C}_{k}^{m \downarrow} \cap \mathcal{V} .
$$




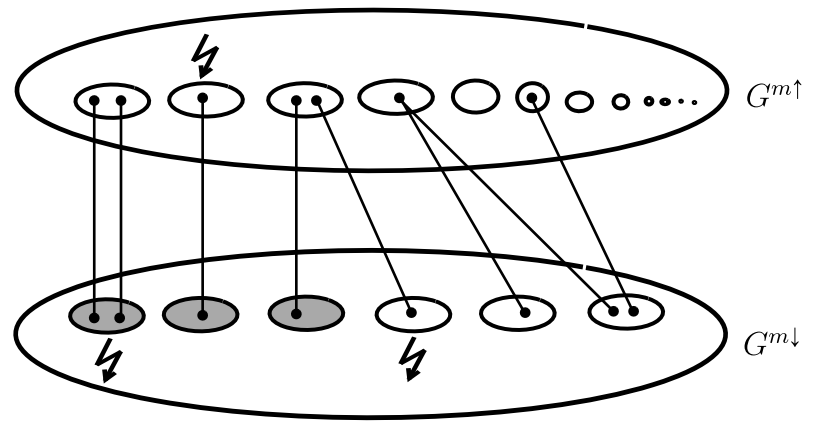

Figure 1. An illustration of Definition 4.6. The blobs marked with a lightning are damaged connected components of $G^{m \downarrow}$ and $G^{m \uparrow}$. The grey blobs are the "bad" components of $G^{m \downarrow}$. The set of indices of "bad" components is denoted by $K^{*}$. Note that intact connected components of $G^{m \downarrow}$ can be "bad" and damaged connected components of $G^{m \downarrow}$ can be "good".

Proof. Let $k \in K \backslash K^{*}$. Denote by $\mathcal{C}^{\prime}$ the connected component of $k$ in $\mathcal{B}$. We prove (4.5) by considering two cases separately.

First case: $k$ is intact.

Denote by $K^{\prime}=\mathcal{C}^{\prime} \cap K$ and $L^{\prime}=\mathcal{C}^{\prime} \cap L$. Then

$$
\mathcal{C}=\left(\bigcup_{k^{\prime} \in K^{\prime}} \mathcal{C}_{k^{\prime}}^{m \downarrow}\right) \cup\left(\bigcup_{l^{\prime} \in L^{\prime}} \mathcal{C}_{l^{\prime}}^{m \uparrow}\right)
$$

is a connected component of $G$ which contains $\mathcal{C}_{k}^{m \downarrow}$ (cf. Definition 4.5), moreover our assumption that $k$ is intact together with $k \in K \backslash K^{*}$ imply that $\mathcal{C}$ is intact (cf. Definition 4.6), thus we have $\mathcal{C}_{k}^{m \downarrow} \cap \mathcal{V}^{(m)}=\mathcal{C}_{k}^{m \downarrow}$ and $\mathcal{C}_{k}^{m \downarrow} \cap \mathcal{V}=\mathcal{C}_{k}^{m \downarrow}$, therefore (4.5) holds.

Second case: $k$ is damaged.

$\mathcal{C}^{\prime} \backslash\{k\}$ is the disjoint union of some connected components $\mathcal{C}_{N}^{\prime}, N \in \mathbb{N}$ of $\mathcal{B} \backslash\{k\}$. Our assumption that $k$ is damaged, Definition 4.6 and the fact that $k \in K \backslash K^{*}$ together imply that there are no parallel edges connected to $k$ in $\mathcal{B}$ and no edge-simple circle of the graph $\mathcal{B}$ contains $k$ as a vertex. Therefore for each $N \in \mathbb{N}$, the cluster $\mathcal{C}_{N}^{\prime}$ is connected to $k$ by one single edge $e_{N}$ of $\mathcal{B}$. Note that $k \in K \backslash K^{*}$ implies that $\mathcal{C}_{N}^{\prime}$ is intact for all $N \in \mathbb{N}$. Therefore, the fires caused by lightnings can only spread "away" from $k$ on the edges $e_{N}, N \in \mathbb{N}$, so by the graphical construction given in Section 3 and Definition 4.3 we obtain (4.5).

Now we define auxiliary random graphs $\hat{G}^{(m)}$ and $\check{G}^{(m)}$ (cf. Lemma 4.4).

Definition 4.8. Let $\check{G}^{(m)}$ be the subgraph of $G$ spanned by the vertices

$$
V\left(\check{G}^{(m)}\right)=\left(\bigcup_{k \in K \backslash K^{*}} \mathcal{C}_{k}^{m \downarrow} \cap \mathcal{V}^{(m)}\right) \cup\left(\bigcup_{k \in K^{*}} \mathcal{C}_{k}^{m \downarrow}\right) \cup\{m+1, m+2, \ldots\}
$$


Define $\hat{G}^{(m)}$ to be the subgraph of $G$ spanned by the vertices

$$
V\left(\hat{G}^{(m)}\right)=\bigcup_{k \in K \backslash K^{*}} \mathcal{C}_{k}^{\downarrow m} \cap \mathcal{V}^{(m)} .
$$

Lemma 4.9. With the above definitions the inclusions (4.2) hold.

Proof. The inclusions $V\left(\hat{G}^{(m)}\right) \subseteq \mathcal{V}^{(m)} \subseteq V\left(\check{G}^{(m)}\right)$ follow from the definitions (4.6), (4.7). Thus $\hat{G}^{(m)} \subseteq H^{(m)} \subseteq \check{G}^{(m)}$ follows from the fact that $H^{(m)}$ is the subgraph of $G$ spanned by the vertex set $\mathcal{V}^{(m)}$.

The inclusions $\hat{G}^{(m)} \subseteq H \subseteq \check{G}^{(m)}$ follow from Lemma 4.7 and the fact that $H$ is the subgraph of $G$ spanned by the vertex set $\mathcal{V}$.

The next lemma is similar to Lemma 2.7.

Lemma 4.10. Given the above set-up let us condition on the graphs $G^{m \downarrow}$ and $G^{m \uparrow}$ and denote by

$$
\alpha=S_{2}^{G^{m \downarrow}}, \quad \beta=S_{2}^{G^{m \uparrow}} .
$$

There exists a constant $C=C(\lambda, t)$ such that if

$$
t^{2} \alpha \beta \leq \frac{1}{2}
$$

holds then we have

$$
\mathbf{E}\left(S_{2}^{\check{G}^{(m)}}-S_{2}^{\hat{G}^{(m)}} \mid G^{m \downarrow}, G^{m \uparrow}\right) \leq C \cdot \beta \cdot\left((1+t \alpha)^{2}+(1+t \alpha) \cdot \alpha^{3 / 2}\right) .
$$

\subsubsection{Proof of Lemma 4.10}

For any subset $\mathcal{C}$ of $\mathbb{N}$, denote by

$$
w(\mathcal{C})=\sum_{i \in \mathcal{C}} m_{i}
$$

the weight of the subset, where $\underline{m}=\left(m_{1}, m_{2}, \ldots\right)$.

Definition 4.11. Define a bipartite weighted graph $\widetilde{\mathcal{B}}$ whose "left" vertices correspond to the connected components of the restriction of $G$ to the vertex set

$$
\widetilde{V}^{(m)}:=V\left(\check{G}^{(m)}\right) \cap\{1, \ldots, m\} \stackrel{(4.6)}{=}\left(\bigcup_{k \in K \backslash K^{*}} \mathcal{C}_{k}^{m \downarrow} \cap \mathcal{V}^{(m)}\right) \cup\left(\bigcup_{k \in K^{*}} \mathcal{C}_{k}^{m \downarrow}\right),
$$

and the "right" vertices correspond to the components of $G^{m \uparrow}$. Define the weights of the vertices of $\widetilde{\mathcal{B}}$ to be the $w(\cdot)$-weight of the corresponding connected components. We declare two vertices in $\widetilde{\mathcal{B}}$ to be connected if the corresponding subsets are connected in $\check{G}^{(m)}$. Denote by $\widetilde{G}^{(m)}$ the subgraph of $G$ spanned by $\widetilde{V}^{(m)}$. 
With the above notation, we have

$$
S_{2}^{\check{G}^{(m)}} \stackrel{(4.6)}{=} S_{2}^{\widetilde{\mathcal{B}}}, \quad S_{2}^{\widetilde{G}^{(m)} \stackrel{(4.7)}{=}} S_{2}^{\hat{G}^{(m)}}+\sum_{k \in K^{*}} w\left(\mathcal{C}_{k}^{m \downarrow}\right)^{2} .
$$

Thus we can start to rewrite the left-hand side of (4.9):

$$
\begin{aligned}
& \mathbf{E}\left(S_{2}^{\check{G}^{(m)}}-S_{2}^{\hat{G}^{(m)}} \mid G^{m \downarrow}, G^{m \uparrow}\right) \\
& =\mathbf{E}\left(S_{2}^{\widetilde{\mathcal{B}}}-S_{2}^{\widetilde{G}^{(m)}} \mid G^{m \downarrow}, G^{m \uparrow}\right)+\mathbf{E}\left(\sum_{k \in K^{*}} w\left(\mathcal{C}_{k}^{m \downarrow}\right)^{2} \mid G^{m \downarrow}, G^{m \uparrow}\right) .
\end{aligned}
$$

In order to show (4.9), it is enough to prove that (4.8) implies

$$
\begin{aligned}
\mathbf{E}\left(S_{2}^{\widetilde{\mathcal{B}}}-S_{2}^{\widetilde{G}^{(m)}} \mid G^{m \downarrow}, G^{m \uparrow}\right) & \leq 2 \beta \cdot(1+t \alpha)^{2}, \\
\mathbf{E}\left(\sum_{k \in K^{*}} w\left(\mathcal{C}_{k}^{m \downarrow}\right)^{2} \mid G^{m \downarrow}, G^{m \uparrow}\right) & \leq 2 t^{2} \lambda \beta \cdot(1+t \alpha) \cdot \alpha^{3 / 2} .
\end{aligned}
$$

First, we deduce (4.10) from Lemma 2.7, with the underlying bipartite graph being $\widetilde{\mathcal{B}}$. Note that the condition (2.14) holds, because $a=S_{2}^{\widetilde{G}^{(m)}} \leq S_{2}^{G^{m \downarrow}}=\alpha$ and $b=S_{2}^{G^{m \uparrow}}=\beta$. Thus we have

$$
\begin{aligned}
& \mathbf{E}\left(S_{2}^{\widetilde{\mathcal{B}}}-S_{2}^{\widetilde{G}^{(m)}} \mid G^{m \downarrow}, G^{m \uparrow},\left(\lambda_{i}\right)_{i=1}^{m}\right) \\
& \quad \stackrel{(2.15)}{\leq} 2 S_{2}^{G^{m \uparrow}} \cdot\left(1+t S_{2}^{\widetilde{G}^{(m)}}\right)^{2} \leq 2 \beta \cdot(1+t \alpha)^{2} .
\end{aligned}
$$

Now (4.10) follows by averaging over the values of $\left(\lambda_{i}\right)_{i=1}^{m}$.

In order to prove (4.11), we first give an upper bound on the probability of the event $\left\{k \in K^{*}\right\}$.

For $k \in K$, denote by $x_{k}^{\prime}=w\left(\mathcal{C}_{k}^{m \downarrow}\right)$ and for $l \in L$, denote $y_{l}^{\prime}=w\left(\mathcal{C}_{l}^{m \uparrow}\right)$. Note that we have

$$
\alpha=\sum_{k \in K}\left(x_{k}^{\prime}\right)^{2}, \quad \beta=\sum_{l \in L}\left(y_{l}^{\prime}\right)^{2} .
$$

Recall the definition of $K^{*}$ from Definition 4.6. The next calculation is similar to (2.9), so we omit the first few steps.

$$
\begin{aligned}
\mathbf{P}(k \in & \left.K^{*} \mid G^{m \downarrow}, G^{m \uparrow}\right) \\
\leq & \sum_{l_{1} \in L}\left(x_{k}^{\prime} y_{l_{1}}^{\prime} t\right)\left(\lambda y_{l_{1}}^{\prime} t\right)+\sum_{l_{1} \in L} \sum_{k_{1} \in K}\left(x_{k}^{\prime} y_{l_{1}}^{\prime} t\right)\left(y_{l_{1}}^{\prime} x_{k_{1}}^{\prime} t\right)\left(\lambda x_{k_{1}}^{\prime} t\right) \\
& \quad+\sum_{l_{1} \in L} \sum_{k_{1} \in K} \sum_{l_{2} \in L}\left(x_{k}^{\prime} y_{l_{1}}^{\prime} t\right)\left(y_{l_{1}}^{\prime} x_{k_{1}}^{\prime} t\right)\left(x_{k_{1}}^{\prime} y_{l_{2}}^{\prime} t\right)\left(\lambda y_{l_{2}}^{\prime} t\right)+\cdots \\
= & x_{k}^{\prime} t^{2} \lambda \beta+x_{k}^{\prime} t^{3} \lambda \alpha \beta+x_{k}^{\prime} t^{4} \lambda \alpha \beta^{2}+\cdots \\
= & x_{k}^{\prime} t^{2} \lambda \beta \cdot(1+t \alpha) \cdot \sum_{n=0}^{\infty}\left(t^{2} \alpha \beta\right)^{n} \stackrel{(4.8)}{\leq} 2 x_{k}^{\prime} t^{2} \lambda \beta \cdot(1+t \alpha) .
\end{aligned}
$$


Now we are ready to prove (4.11):

$$
\begin{aligned}
\mathbf{E}\left(\sum_{k \in K^{*}}\left(x_{k}^{\prime}\right)^{2} \mid G^{m \downarrow}, G^{m \uparrow}\right) & \leq \sum_{k \in K} 2\left(x_{k}^{\prime}\right)^{3} t^{2} \lambda \beta \cdot(1+t \alpha) \\
& \stackrel{(*)}{\leq} 2 t^{2} \lambda \beta \cdot(1+t \alpha) \cdot \alpha^{3 / 2},
\end{aligned}
$$

where in $(*)$ we used the fact that $x_{k}^{\prime} \leq \sqrt{\alpha}$ for any $k \in K$. This completes the proof of (4.9) and Lemma 4.10.

\subsection{Proof of Theorem 4.2}

Let us fix $t, \lambda \in \mathbb{R}_{+}$, the sequence $\underline{m}^{(n)}, n \in \mathbb{N}$ and the limit $\underline{m}^{(\infty)}$. For any $n \in \mathbb{N}_{+} \cup\{\infty\}$, let $\mathbf{m}_{t}^{(n, m)}$ denote the realization under the $(\xi, \lambda)$-coupling of the $\operatorname{MCLD}(\lambda)$ with initial state

$$
\underline{m}^{(n, m)}=\left(m_{1}^{(n)}, \ldots, m_{m}^{(n)}, 0,0, \ldots\right), \quad \text { where } \underline{m}^{(n)}=\left(m_{1}^{(n)}, m_{2}^{(n)}, \ldots\right) .
$$

We also define $\mathcal{V}_{t}^{(n, m)}$ to be the set of intact vertices of the graph $H_{t}^{(n, m)}$ of the $\operatorname{MCLD}(\lambda)$ with initial state $\underline{m}^{(n, m)}$ under the $(\xi, \lambda)$-coupling.

In order to prove (4.1), we only need to show that for every $\varepsilon>0$ there exists $m, n_{0} \in \mathbb{N}$ such that for all $n \geq n_{0}$ we have

$$
\begin{aligned}
& \mathbf{P}\left(\mathrm{d}\left(\mathbf{m}_{t}^{(n)}, \mathbf{m}_{t}^{(n, m)}\right)\right.\geq \varepsilon) \leq 4 \varepsilon, \\
& \mathbf{P}\left(\mathrm{d}\left(\mathbf{m}_{t}^{(n, m)}, \mathbf{m}_{t}^{(\infty, m)}\right) \geq \varepsilon\right) \leq \varepsilon, \\
& \mathbf{P}\left(\mathrm{d}\left(\mathbf{m}_{t}^{(\infty, m)}, \mathbf{m}_{t}^{(\infty)}\right) \geq \varepsilon\right) \leq 4 \varepsilon .
\end{aligned}
$$

Let us fix $\varepsilon>0$. We know from Lemma 2.4 that

$$
\mathbf{P}\left(S_{2}^{G_{t}^{(\infty)}}<+\infty\right)=1
$$

where $G_{t}^{(\infty)}$ denotes the random graph constructed from the exponential random variables $\left(\xi_{i, j}\right)_{1 \leq i<j<\infty}$ and the initial state $\underline{m}^{(\infty)} \in \ell_{2}^{\downarrow}$ according to the rules described in Definition 2.3. Given $\varepsilon>0$, we can find $M \in \mathbb{R}_{+}$such that

$$
\mathbf{P}\left(S_{2}^{G_{t}^{(\infty)}} \geq M-1\right) \leq \varepsilon
$$

Recall the notion of the constant $C=C(t, \lambda)$ from Lemma 4.10. Let us choose $\delta>0$ such that

$$
t^{2} M \delta \leq \frac{1}{2} \quad \text { and } \quad 9 C \cdot \delta \cdot\left((1+t M)^{2}+(1+t M) \cdot M^{3 / 2}\right) \leq \varepsilon^{3}
$$

Now we choose the truncation threshold $m$. Since $\underline{m}^{(n)} \rightarrow \underline{m}^{(\infty)}$ in $l_{2}$, we can make

$$
\sup _{n \in \mathbb{N} \cup\{\infty\}}\left\|\underline{m}^{(n)}-\underline{m}^{(n, m)}\right\|_{2}
$$


(where $\underline{m}^{(n, m)}$ is defined in (4.12)) as small as we wish by making $m$ large. Thus by (2.11) and the Markov inequality we can choose $m$ such that

$$
\sup _{n \in \mathbb{N} \cup\{\infty\}} \mathbf{P}\left(S_{2}^{G_{t}^{(n, m) \uparrow}} \geq \delta\right) \leq \varepsilon .
$$

Having fixed $m$, we note that under the $(\xi, \lambda)$-coupling we have

$$
\mathrm{d}\left(\mathbf{m}_{t}^{(n, m)}, \mathbf{m}_{t}^{(\infty, m)}\right) \stackrel{p}{\longrightarrow} 0, \quad n \rightarrow \infty
$$

We also have

$$
S_{2}^{G_{t}^{(n, m) \downarrow}} \stackrel{p}{\longrightarrow} S_{2}^{G_{t}^{(\infty, m) \downarrow}} \leq S_{2}^{G_{t}^{(\infty)}}
$$

thus we can choose $n_{0}$ such that for all $n \geq n_{0}$ we have (4.14) and

$$
\forall n \in\left\{n_{0}, n_{0}+1, \ldots\right\} \cup\{\infty\}: \mathbf{P}\left(S_{2}^{G_{t}^{(n, m) \downarrow}} \geq M\right) \stackrel{(4.16),(4.19)}{\leq} 2 \varepsilon .
$$

We are ready to show (4.13) and (4.15) for the above choice of $m$ and $n_{0}$.

For any $n \in\left\{n_{0}, n_{0}+1, \ldots\right\} \cup\{\infty\}$ we have

$$
\begin{gathered}
\mathbf{P}\left(\mathrm{d}\left(\mathbf{m}_{t}^{(n)}, \mathbf{m}_{t}^{(n, m)}\right) \geq \varepsilon\right) \leq \quad \mathbf{P}\left(S_{2}^{G_{t}^{(n, m) \uparrow}} \geq \delta\right)+\mathbf{P}\left(S_{2}^{G_{t}^{(n, m) \downarrow}} \geq M\right) \\
+\mathbf{P}\left(\mathrm{d}\left(\mathbf{m}_{t}^{(n)}, \mathbf{m}_{t}^{(n, m)}\right) \geq \varepsilon, S_{2}^{G_{t}^{(n, m) \uparrow}} \leq \delta, S_{2}^{G_{t}^{(n, m) \downarrow}} \leq M\right) \\
\stackrel{(4.18),(4.20)}{\leq} \varepsilon+2 \varepsilon+\mathbf{P}\left(\mathrm{d}\left(\mathbf{m}_{t}^{(n)}, \mathbf{m}_{t}^{(n, m)}\right) \geq \varepsilon, A\right),
\end{gathered}
$$

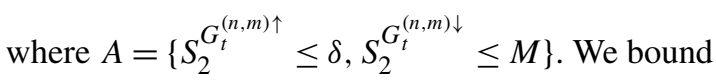

$$
\begin{aligned}
\mathbf{P}\left(\mathrm{d}\left(\mathbf{m}_{t}^{(n)}, \mathbf{m}_{t}^{(n, m)}\right) \geq \varepsilon, A\right) & \stackrel{(4.3)}{\leq} \mathbf{P}\left(9 \cdot\left(S_{2}^{\check{G}^{(n, m)}}-S_{2}^{\hat{G}^{(n, m)}}\right) \geq \varepsilon^{2}, A\right) \\
& =\mathbf{E}\left(\mathbf{P}\left(9 \cdot\left(S_{2}^{\check{G}^{(n, m)}}-S_{2}^{\hat{G}^{(n, m)}}\right) \geq \varepsilon^{2} \mid G^{(n, m) \downarrow}, G^{(n, m) \uparrow}\right) ; A\right) \\
& \stackrel{(*)}{\leq} \frac{9 C \cdot \delta \cdot\left((1+t M)^{2}+(1+t M) \cdot M^{3 / 2}\right)}{\varepsilon^{2}} \stackrel{(4.17)}{\leq} \varepsilon,
\end{aligned}
$$

where in the equation marked by $(*)$ we used Lemma 4.10 and the Markov inequality. This concludes the proof of (4.13), (4.14), (4.15) and Theorem 4.2.

\section{Acknowledgements}

I thank James Martin for collaborating with me on [15], which inspired this work. I also thank an anonymous referee for useful comments on the manuscript. This work is partially supported by OTKA (Hungarian National Research Fund) grant K100473, the Postdoctoral Fellowship of the Hungarian Academy of Sciences and the Bolyai Research Scholarship of the Hungarian Academy of Sciences. 


\section{References}

[1] Addario-Berry, L., Broutin, N. and Goldschmidt, C. (2012). The continuum limit of critical random graphs. Probab. Theory Related Fields 152 367-406. MR2892951

[2] Ahlberg, D., Duminil-Copin, H., Kozma, G. and Sidoravicius, V. (2015). Seven-dimensional forest fires. Ann. Inst. Henri Poincaré Probab. Stat. 51 862-866. MR3365964

[3] Aldous, D. (1997). Brownian excursions, critical random graphs and the multiplicative coalescent. Ann. Probab. 25 812-854. MR1434128

[4] Aldous, D. and Limic, V. (1998). The entrance boundary of the multiplicative coalescent. Electron. J. Probab. 3 No. 3, 59 pp. MR1491528

[5] Aldous, D.J. (2000). The percolation process on a tree where infinite clusters are frozen. Math. Proc. Cambridge Philos. Soc. 128 465-477. MR1744108

[6] Bhamidi, S., Broutin, N., Sen, S. and Wang, X. (2014). Scaling limits of random graph models at criticality: Universality and the basin of attraction of the Erdős-Rényi random graph. Available at arXiv:1411.3417.

[7] Bhamidi, S., van der Hofstad, R. and Sen, S. (2015). The multiplicative coalescent, inhomogeneous continuum random trees, and new universality classes for critical random graphs. Available at arXiv:1508.04645.

[8] Bhamidi, S., van der Hofstad, R. and van Leeuwaarden, J.S.H. (2010). Scaling limits for critical inhomogeneous random graphs with finite third moments. Electron. J. Probab. 15 1682-1703. MR2735378

[9] Bhamidi, S., van der Hofstad, R. and van Leeuwaarden, J.S.H. (2012). Novel scaling limits for critical inhomogeneous random graphs. Ann. Probab. 40 2299-2361. MR3050505

[10] Dürre, M. (2006). Existence of multi-dimensional infinite volume self-organized critical forest-fire models. Electron. J. Probab. 11 513-539. MR2242654

[11] Dürre, M. (2006). Uniqueness of multi-dimensional infinite volume self-organized critical forest-fire models. Electron. Commun. Probab. 11 304-315. MR2266720

[12] Kiss, D. (2015). Frozen percolation in two dimensions. Probab. Theory Related Fields 163 713-768. MR3418754

[13] Kiss, D., Manolescu, I. and Sidoravicius, V. (2015). Planar lattices do not recover from forest fires. Ann. Probab. 43 3216-3238. MR3433580

[14] Limic, V. (1998). Properties of the multiplicative coalescent. Ph.D. thesis, UC Berkeley.

[15] Martin, J.B. and Ráth, B. Rigid representations of the multiplicative coalescent with linear deletion. Electron. J. Probab. To appear. Available at arXiv:1610.00891.

[16] Martin, J.B. and Ráth, B. Scaling limits of mean-field frozen percolation and forest fire processes (in preparation).

[17] Merle, M. and Normand, R. (2014). Self-organized criticality in a discrete model for Smoluchowski's equation. Available at arXiv: 1410.8338.

[18] Ráth, B. (2009). Mean field frozen percolation. J. Stat. Phys. 137 459-499. MR2564286

[19] Ráth, B. and Tóth, B. (2009). Erdôs-Rényi random graphs + forest fires = self-organized criticality. Electron. J. Probab. 14 1290-1327. MR2511285

[20] van den Berg, J., de Lima, B.N.B. and Nolin, P. (2012). A percolation process on the square lattice where large finite clusters are frozen. Random Structures Algorithms 40 220-226. MR2877564

[21] van den Berg, J., Kiss, D. and Nolin, P. (2015). Two-dimensional volume-frozen percolation: Deconcentration and prevalence of mesoscopic clusters. Available at arXiv:1512.05335.

[22] van den Berg, J. and Nolin, P. (2017). Two-dimensional volume-frozen percolation: Exceptional scales. Ann. Appl. Probab. 27 91-108. MR3619783 
[23] van den Berg, J. and Tóth, B. (2001). A signal-recovery system: Asymptotic properties, and construction of an infinite-volume process. Stochastic Process. Appl. 96 177-190. MR1865354

Received October 2016 and revised July 2017 